\title{
Confining strings, infinite statistics, and integrability
}

\author{
John C. Donahue@ and Sergei Dubovsky® \\ Center for Cosmology and Particle Physics, Department of Physics, New York University,
} New York, New York 10003, USA

(Received 2 August 2019; accepted 20 March 2020; published 21 April 2020)

\begin{abstract}
We study confining strings in massive adjoint two-dimensional chromodynamics. Off shell, as a consequence of zigzag formation, the resulting world sheet theory provides a nontrivial dynamical realization of infinite quon statistics. Taking the high energy limit we identify a remarkably simple and novel integrable relativistic $N$-body system. Its symmetry algebra contains an additional "shadow" Poincaré subalgebra. This model describes the $N$-particle subsector of a $T \bar{T}$-deformed massless fermion.
\end{abstract}

DOI: 10.1103/PhysRevD.101.081901

\section{INTRODUCTION}

$S U\left(N_{c}\right)$ quantum chromodynamics (QCD) turns into a free string theory in the planar $\left(N_{c} \rightarrow \infty\right)$ limit [1]. For its maximally supersymmetric cousin the corresponding string theory has been identified as type IIB critical superstrings on $\mathrm{AdS}_{5} \times S_{5}$ [2]. Moreover, the corresponding world sheet theory is integrable and has been solved, resulting in the exact spectrum of planar $\mathcal{N}=4$ Yang-Mills (YM) (see [3] for an overview).

It has proven excruciatingly difficult to reproduce this success for planar nonsupersymmetric gluodynamics. The corresponding string theory has not even been identified yet, despite a rich 45 year-long history of study. One may suspect at first that the question is not sufficiently well posed. However, a sharp version of the problem can be formulated as follows [4]. Consider a background of YM theory with a single infinitely long confining string (flux tube). In the strict planar limit the string excitations decouple from bulk degrees of freedom and give rise to a microscopic two-dimensional model. The challenge is to build this world sheet theory.

With this sharp formulation at hand, one immediately understands the source of the difficulties. Namely, in the absence of additional massless degrees of freedom on the world sheet, the flux tube theory in $D=4$-dimensional space-time has irreducible particle production [5,6], associated with the Polchinski-Strominger term [7]. Lattice YM simulations [8-13] (see [14,15] for reviews) rule out the presence of additional massless excitations on the world sheet, thus excluding integrability.

Published by the American Physical Society under the terms of the Creative Commons Attribution 4.0 International license. Further distribution of this work must maintain attribution to the author(s) and the published article's title, journal citation, and DOI. Funded by SCOAP.
Note that at $D=3$ integrability does not require additional degrees of freedom [4]. However, a closer look at the lattice data shows that the world sheet theory is not integrable in $D=3$ YM either [16-18].

Hence, unlike for the $\mathcal{N}=4$ case, in the planar limit of nonsupersymmetric YM we are left with an interacting nonintegrable two-dimensional model. To make life harder (and more interesting), at high energies this model exhibits characteristically gravitational behavior instead of that of a conventional quantum field theory $[19,20]$.

This leaves two directions for further progress. First, as gluodynamics does not have any relevant deformations, the world sheet theory is likely isolated. This turns it into a natural target for the modern $S$-matrix bootstrap [21,22], following the success of the conformal bootstrap in describing another isolated theory-the $3 D$ Ising model [23]. A first promising step in this direction has been made very recently [24].

The other possibility is to identify a nearby integrable model, and to obtain a description of the world sheet dynamics by performing a systematic perturbative expansion around this integrable theory. The quality of the latter expansion will then provide a natural measure of the proximity of the integrable mode to the full world sheet theory.

One may object that there is no a priori reason for a controlled integrable approximation to exist. However, the analysis of lattice data provides a number of tantalizing hints supporting this program. This motivated a proposal for an integrable approximation-the axionic string ansatz (ASA) — both at $D=3$ and $D=4[4,17,25,26]$.

The successes of the ASA have a clear physical origin $[20,27]$. At low energies the world sheet degrees of freedom are translational Goldstone bosons of the nonlinearly realized Poincaré symmetry [5,28-32]. Their low energy dynamics is well approximated by a classically integrable Nambu-Goto action. On the other hand, at high energies world sheet degrees of freedom correspond to partons of 
perturbative QCD (gluons in the YM case). Asymptotic freedom then implies that hard particle production is suppressed also at high energies.

This reasoning suggests that the violation of world sheet integrability may be a transient phenomenon dominated by intermediate energies, $E \sim \Lambda_{\mathrm{QCD}}$. A combination of low and high energy expansions may then allow one to describe the world sheet dynamics at all scales.

As argued in [20], a natural playground to test these ideas is provided by adjoint $D=2 \mathrm{QCD}$. As the world sheet theory lives in two dimensions for any $D$, general lessons from the study of the world sheet should be universally applicable.

The goal of the present paper is to report a solution to the very first step in this program-the identification of an integrable approximation at $D=2$.

\section{HAMILTONIAN FORMALISM AND INFINITE STATISTICS}

Massive adjoint $\mathrm{QCD}_{2}$ is defined by the following action:

$$
S=\int d \tau d \sigma \operatorname{Tr}\left(\frac{-1}{2 g^{2}} F_{\mu \nu} F^{\mu \nu}+\bar{\psi}\left(i \mathbb{\nabla}^{(a d)}-m\right) \psi\right),
$$

where $\psi$ is a Majorana fermion in the adjoint representation of the $S U\left(N_{c}\right)$ gauge group. ${ }^{1}$ We will mostly consider the heavy mass regime

$$
m^{2} \gg g^{2} N_{c},
$$

where one expects a straightforward perturbative treatment to apply. The spectrum of this model was studied extensively in early 1990s [34-36] (see [37] for a recent update). A study of the world sheet dynamics has been initiated in [20], we will adopt the same procedure here.

Following [38,39], we put the theory on a finite interval, $\sigma \in(-L, L)$ with an infinitely heavy fundamental quarkantiquark pair $Q, \bar{Q}$ placed at the end points. Eventually, we take the infinite volume limit $L \rightarrow \infty$. Then the world sheet theory describes dynamics of states created by gauge invariant single-trace operators of the form

$$
\mathcal{O}_{w}=\bar{Q}\left(P e^{i \int_{\mathcal{C}} d \sigma A_{\sigma}} \psi\left(\sigma_{1}\right) \ldots \psi\left(\sigma_{N}\right)\right) Q .
$$

Here the integration path $\mathcal{C}$ starts at $\sigma=-L$ and ends at $\sigma=L$, but does not have to be straight. Turning points are allowed at the locations $\sigma_{i}$ of the adjoint quarks insertions because the Polyakov zigzag symmetry [40] is broken in the presence of the adjoint matter. Physically, one may think that the world sheet theory describes the interior of a heavy quark $Q \bar{Q}$ meson.

\footnotetext{
${ }^{1}$ A study of different fermion representations can be found in, e.g., [33].
}

After fixing the spatial gauge, $A_{\sigma}=0$, one can integrate out the remaining nondynamical component $A_{\tau}$ of the gauge field. The resulting Hamiltonian acts in the extended Hilbert space

$$
\mathcal{H}_{\text {ex }}=V \otimes \mathcal{H}_{f} \otimes \bar{V}
$$

where $\mathcal{H}_{f}$ is the free fermion Fock space, and $V(\bar{V})$ are (anti)fundamental representations of the color group. These additional factors represent color degrees of freedom of the end point quarks $Q(\bar{Q})$. As a consequence of confinement, the physical Hilbert space $\mathcal{H}_{\mathrm{ph}}$ is the subspace of $\mathcal{H}_{\mathrm{ex}}$ annihilated by all color charges,

$$
\left(\mathcal{T}^{a}+\overline{\mathcal{T}}^{a}+\int d \sigma \rho^{a}\right) \mathcal{H}_{\mathrm{ph}}=0 .
$$

Here $\rho^{a}$ is the color density of adjoint fermions, and $\mathcal{T}^{a}\left(\overline{\mathcal{T}}^{a}\right)$ are (anti)fundamental generators representing color charges of $Q(\bar{Q})$.

To describe the physical states it is convenient to adopt operator notations following from the identification

$$
V \otimes \bar{V}=L(V)
$$

where $L(V)$ is the space of linear operators acting on $V$. Then a general state in $\mathcal{H}_{\text {ex }}$ takes the form

$$
|\psi\rangle=\sum_{i}\left|\psi_{i}\right\rangle_{F} \otimes M_{i},
$$

with $\left|\psi_{i}\right\rangle_{F} \in \mathcal{H}_{f}$ and $M_{i} \in L(V)$. The physical world sheet Hilbert space $\mathcal{H}_{w}$ is generated by color singlets of the form

$$
\mathcal{H}_{w}=\left\{\psi^{a_{1}} \cdots \psi^{a_{N}}|0\rangle_{F} \otimes T^{a_{1}} \cdots T^{a_{N}}\right\} .
$$

Here $T^{a}$ 's are fundamental $S U\left(N_{c}\right)$ generators considered as elements of $L(V)$. These are not to be confused with the quantum operators $\mathcal{T}^{a}$ 's in (5). Note that $\mathcal{H}_{p h}$ contains additional multitrace color singlet states such as the mesonic state $\psi^{a} \psi^{a}|0\rangle_{F} \otimes \mathbb{1}$. Multitrace states decouple from the world sheet in the planar limit.

In the momentum representation the physical world sheet states can be written as linear combinations of

$\left|k_{1}, \ldots, k_{N}\right\rangle=\frac{1}{\sqrt{2}}\left(\frac{2}{N_{c}}\right)^{\frac{N+1}{2}} \prod_{i=1}^{N} b_{k_{i}}^{a_{i} \dagger}|0\rangle_{F} \otimes \prod_{i=1}^{N} T^{a_{i}}$,

where $b_{k}^{a \dagger}$ are fermionic creation operators. For many purposes it is convenient to also use the coordinate representation. The corresponding basis is

$$
\left|\sigma_{1}, \ldots, \sigma_{N}\right\rangle=\int \prod_{i=1}^{N} \frac{d k_{i}}{\sqrt{2 \pi}} e^{-i k_{i} \sigma_{i}}\left|k_{1}, \ldots, k_{N}\right\rangle .
$$


In the heavy mass regime (2) eigenstates of the world sheet Hamiltonian are well characterized by their parton number. In particular, one finds the world sheet vacuum $|0\rangle=$ $|0\rangle_{F} \otimes \mathbb{1}$ and one-particle excitations $|k\rangle=\frac{\sqrt{2}}{N_{c}} b_{k}^{a \dagger} \otimes T^{a}$, dubbed "free quarks" in [39].

As emphasized in [20], the world sheet multiparticle states (9) and (10) do not describe conventional identical particles. For instance, the configuration space of twoparticle states (10) is the whole plane instead of the halfplane, as $\left|\sigma_{1}, \sigma_{2}\right\rangle \neq\left|\sigma_{2}, \sigma_{1}\right\rangle$. Equivalently, in the planar limit the exchange term is missing in multiparticle inner products,

$$
\left\langle\sigma_{N}^{\prime}, \ldots, \sigma_{1}^{\prime} \mid \sigma_{1}, \ldots, \sigma_{N}\right\rangle=\prod_{i=1}^{N} \delta\left(\sigma_{i}-\sigma_{i}^{\prime}\right) .
$$

This is the inner product for a system of $N$ distinguishable particles, indicating that the world sheet theory provides a nontrivial dynamical realization of infinite quon statistics (see, e.g., [41]). It also serves as an interesting counterexample to the common lore [42] that the conventional Fock space is the only possible arena for Lorentz invariant quantum dynamics.

Up to now our treatment of the world sheet theory was mostly kinematical. To study dynamics and to implement the program outlined in the Introduction we need to evaluate how the world sheet Hamiltonian acts on the physical space $\mathcal{H}_{w}$ and to learn how to develop perturbation theory in this unconventional Hilbert space. We leave this task for a separate publication [43] and will discuss here only the very first step-identification of an unperturbed Hamiltonian $H$.

Let us first inspect two-particle matrix elements of the full world sheet Hamiltonian $H_{w}$. In the c.o.m. frame one finds [20]

$$
\left\langle k_{2}, k_{1}\left|H_{w}\right| k,-k\right\rangle=\delta\left(k_{1}+k_{2}\right)\left(\delta\left(k_{1}-k\right) 2 \omega_{k}-\frac{g^{2} N_{c}}{4 \pi} \mathcal{V}\right) .
$$

Here $\omega_{k}=\sqrt{k^{2}+m^{2}}$ and

$\mathcal{V}=\mathcal{U}\left(k, k_{1}\right) \frac{\mathcal{P}}{\left(k-k_{1}\right)^{2}}+i \pi \delta^{\prime}\left(k-k_{1}\right)-\frac{m^{2}}{4 \omega_{k}^{2} \omega_{k_{1}}^{2}}$,

where $\mathcal{U}\left(k, k_{1}\right)$ is a smooth function of momenta. It is equal to unity for $k, k_{1} \ll m$, or $k, k_{1} \gg m$ and also in the forward limit $k=k_{1}$. $\mathcal{P}$ stands for the principal value.

Even though $\mathcal{V}$ is multiplied by the 't Hooft coupling, it cannot be entirely treated as a perturbation due to the presence of forward singularities in (12). The physical meaning of these singularities is transparent in position space. Setting $\mathcal{U}\left(k, k_{1}\right)=1$, dropping the last nonsingular term in (12) and switching to position space we arrive at

$$
\mathcal{V}_{0}(\sigma)=\sigma+|\sigma|
$$

We see that the conventional statistics gets restored onshell- the growth of the potential (13) at $\sigma=+\infty$ reduces the number of scattering states by a factor of 2 , restoring the agreement with state counting for conventional identical particles.

It is straightforward to check that the story repeats for multiparticle states. Namely, all terms in the full Hamiltonian which grow at spatial infinity are diagonal in the particle number and combine into the following potential in the $N$-particle sector:

$$
V_{N}=\frac{g^{2} N_{c}}{4 \pi} \sum_{i=1}^{N-1} \mathcal{V}_{0}\left(\sigma_{i, i+1}\right),
$$

where $\sigma_{i, i+1}=\sigma_{i}-\sigma_{i+1}$. As a result, in the asymptotic regions $\tau \rightarrow \pm \infty$ one only finds configurations with $\sigma_{1} \leq$ $\sigma_{2} \cdots \leq \sigma_{N}$ and the conventional statistics gets restored on shell for any number of colliding particles. Zigzags responsible for the emergence of the off-shell infinite statistics cost energy and do not survive on shell.

\section{INTEGRABLE WORLD SHEET MECHANICS}

These results suggest the following choice of the unperturbed Hamiltonian in the world sheet perturbation theory:

$$
H_{N, m}=\sum_{i=1}^{N} \sqrt{p_{i}^{2}+m^{2}}+V_{N},
$$

where the subscript $N$ indicates that (15) is a restriction of the unperturbed Hamiltonian $H$ to the $N$-particle sector. In addition to the conventional free piece, $H_{N, m}$ also incorporates the leading forward singularities, which determine the asymptotic growth of the potential. A similar leading order Hamiltonian was also obtained in the Abelian case (at $N=2$ ) based on $\hbar$ counting [38].

To see whether (15) is a good choice it is natural to check at least the following two conditions:

(i) Is (15) Poincaré invariant?

(ii) Is (15) integrable?

We restrict to the classical analysis of (15). Even though the procedure which lead us to (15) was not manifestly Lorentz covariant, the final result is. Indeed, piecewise the potential (14) is either free or describes a constant electric field acting on some of the particles. Both options correspond to Lorentz invariant dynamics in two dimensions. More precisely, a single particle moving in a constant electric field is invariant under the centrally extended Poincaré group [44]. However, the central charge vanishes for (15) after contributions from all particles are added up. The corresponding boost generator is

$$
J=\sum_{i=1}^{N} \sigma_{i} \sqrt{p_{i}^{2}+m^{2}}+\frac{1}{2} \sum_{i=1}^{N-1}\left(\sigma_{i}+\sigma_{i+1}\right) \mathcal{V}_{0}\left(\sigma_{i, i+1}\right) .
$$



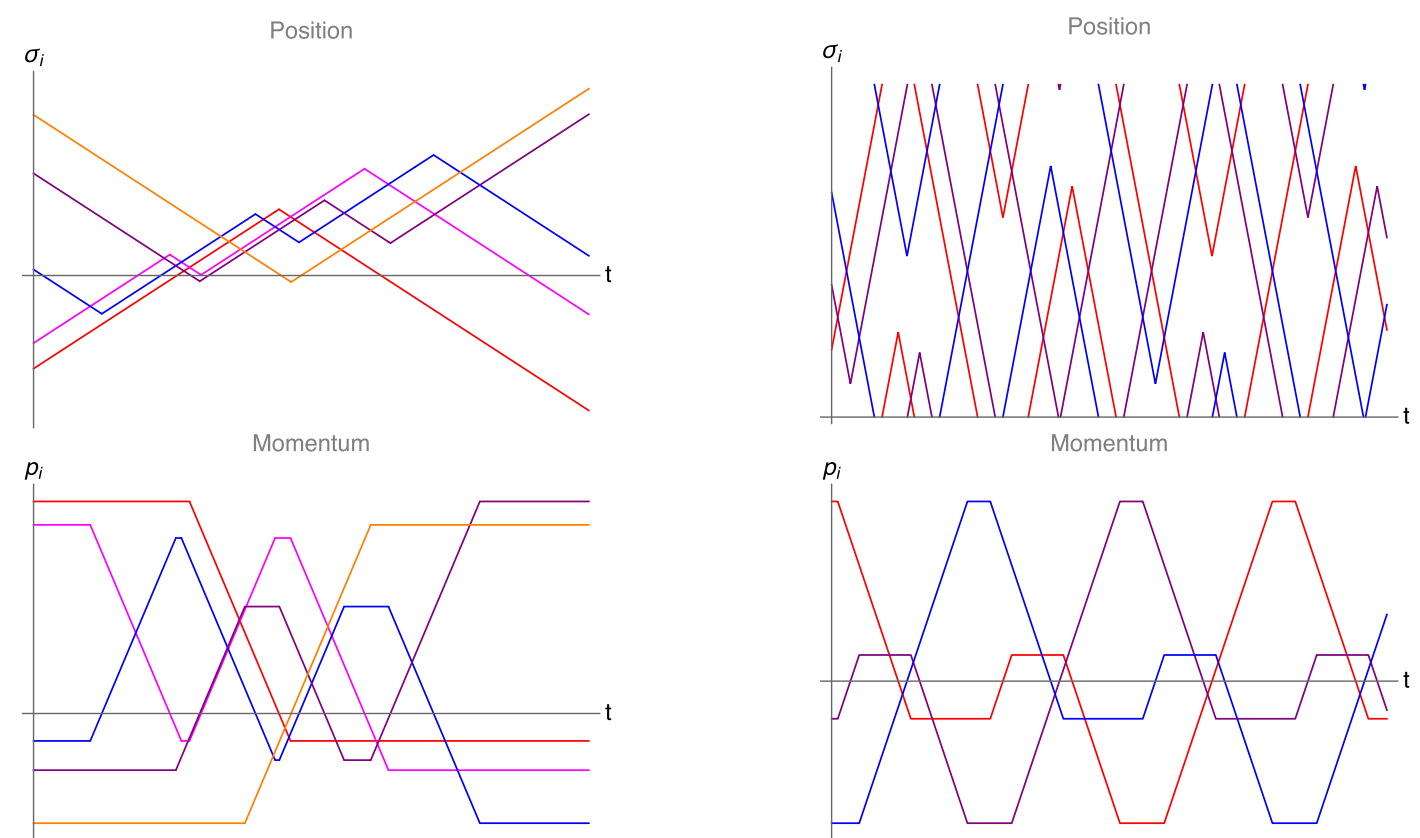

FIG. 1. Time evolution for a sample five-particle scattering (left) and of a three-particle configuration in the periodic case (right).

The Poisson brackets between $H, J$ and the total momentum $P$ satisfy the $I S O(1,1)$ Poincaré algebra

$$
\{H, P\}=0, \quad\{J, P\}=H, \quad\{J, H\}=P .
$$

One might expect then that the system (15) is also integrable, i.e., no momentum transfer occurs in multiparticle collisions. Indeed, as a consequence of crossing symmetry, momentum transfer implies particle production. The latter is absent for $H$. In accord with this argument, in the relativistic two-dimensional ${ }^{2} \mathrm{~N}$-body systems of $[48,49]$ integrability automatically follows from Poincaré invariance.

However, a straightforward Mathematica simulation of (15) shows that the system is not integrable. Most likely this is related to difficulties with preserving the Poincare algebra (17) at the quantum level in very similar models $[50,51]$. We leave it to [43] to study whether this may be resolved by including subleading forward singularities in $H$. Instead, here we observe that the high energy limit of (15) given by

$$
H_{N} \equiv H_{N, 0}=\sum_{i=1}^{N}\left|p_{i}\right|+\sum_{i=1}^{N-1}\left(\sigma_{i, i+1}+\left|\sigma_{i, i+1}\right|\right)
$$

does give rise to a Poincaré invariant integrable $N$-body model. From now on we set the 't Hooft coupling to unity, $g^{2} N_{c}=4 \pi$. Note that this limit does not contradict (2), as

\footnotetext{
${ }^{2}$ Recall that at $D>2$ the "no interaction theorem" [45-47] excludes interacting finite-dimensional relativistic Hamiltonian systems.
}

$p^{2} \gg m^{2} \gg g^{2} N_{c}$. On the other hand, (18) does not actually rely on (2) - this Hamiltonian describes the high energy world sheet dynamics independently of whether (2) holds or not.

Integrability of (18) is the main observation of this paper. It confirms that (18) does provide a good starting point for the high energy expansion on the world sheet. The fastest way to check integrability is to solve the equations of motion,

$$
\dot{\sigma}_{i}=s_{i}, \quad \dot{p}_{i}=s_{i-1, i}-s_{i, i+1} .
$$

Here $s_{i}=\operatorname{sign} p_{i}, \quad s_{0,1}=s_{N, N+1}=-1 \quad$ and $\quad s_{j, j+1}=$ $\operatorname{sign} \sigma_{j, j+1}$ for $1 \leq j<N$. A general solution of (19) is a piecewise linear function of time. Using Mathematica ${ }^{3}$ it does not take long to convince oneself that the initial and final asymptotic sets of momenta are always the same. As an illustration, in Fig. 1 we plot a nontrivial five-particle collision and a self-repeating three-particle solution of the integrable periodic version of the model obtained by setting $s_{0,1}=s_{N, N+1}=\operatorname{sign}\left(\sigma_{N}-\sigma_{1}-2 \pi\right)$.

We will present a detailed study of the resulting integrable structure in [43] and restrict here to just a few remarks. Given the piecewise linear time dependence of the solutions, it is natural to look for conserved topological invariants $T(S)$ and also for stepwise linear conserved charges of the form

$$
I(\sigma, p)=A^{i}(S) \sigma_{i}+B^{i}(S) p_{i},
$$

\footnotetext{
${ }^{3}$ An exact Mathematica solver and the resulting movies can be downloaded at https://jcdonahue.net/research.
} 
where $S=\left(s_{0,1}, s_{1}, \ldots, s_{n}, s_{n, n+1}\right)$ is the set of all signs. For instance, using (19) it is straightforward to check that

$$
T_{2}=\frac{1}{2} \sum_{i=1}^{N} s_{i}\left(s_{i-1, i}+s_{i, i+1}\right)
$$

stays constant. To see the physical meaning of $T_{2}$ let us evaluate it in the asymptotic regions, where all $s_{i-1, i}=-1$. One finds

$$
T_{2}=N_{L}-N_{R},
$$

where $N_{L(R)}$ are the numbers of left(right) movers in the asymptotic regions. It is natural to think of $S$ as a sequence of classical spin variables. Interestingly, $T_{2}$ turns then into the Ising Hamiltonian.

$T_{2}$ also provides a convenient starting point to construct a nontopological charge by looking for $F_{T_{2}}$ satisfying $\left\{F_{T_{2}}, H\right\}=T_{2}$. Using (19) it is straightforward to construct $F_{T_{2}}$ in the nonperiodic case,

$$
F_{T_{2}}=\sum_{k=1}^{N}\left(k-\frac{1}{2}\right)\left|p_{k}\right|+\sum_{k=1}^{N-1} k\left|\sigma_{k, k+1}\right|-N\left(\sigma_{N}+\frac{H_{N}}{2}\right) .
$$

$F_{T_{2}}$ itself is only conserved in the sector with $N_{L}=N_{R}$. However, it is possible to build a new conserved quantity in all sectors by acting on $F_{T_{2}}$ with the boost generator. Namely, let us define

$$
\tilde{P}=T_{2}\left\{J, F_{T_{2}}\right\}-N F_{T_{2}}, \quad \tilde{H}=T_{2} F_{T_{2}}-N\left\{J, F_{T_{2}}\right\} .
$$

Then the Poincaré algebra (17) gets enlarged to

$$
\begin{aligned}
\{\tilde{H}, \tilde{P}\} & =0, \quad\{J, \tilde{P}\}=\tilde{H}, \quad\{J, \tilde{H}\}=\tilde{P} \\
\{H, \tilde{H}\} & =\{\tilde{P}, P\}=N^{2}-T_{2}^{2}, \quad\{P, \tilde{H}\}=\{H, \tilde{P}\}=0 .
\end{aligned}
$$

It was suggested in [20] that in a putative gravitational description of the world sheet the zigzags should correspond to black holes and the exotic off-shell statistics to black hole complementarity. In this context it is encouraging to find the "shadow" Poincaré subalgebra $(\tilde{H}, \tilde{P}, J)$. It will be interesting to check whether the shadow charges $(\tilde{H}, \tilde{P})$ may be identified with the Hamiltonian and momentum seen by infalling observers.

Similar techniques also allow one to obtain additional integrals in involution at $N>2$. For instance, for $N=3$ the following translationally invariant combination is conserved in the $N_{R}=2, N_{L}=1$ sector

$$
I_{12}=\mathcal{V}_{0}\left(p_{1}\right)+\mathcal{V}_{0}\left(\sigma_{1,2}\right)+\frac{\mathcal{V}_{0}\left(p_{2}\right)}{4}\left(3+s_{1}\left(s_{1,2}-1\right)+s_{1,2}\right)
$$

This establishes Liouville integrability at $N=3$. Interestingly, $I_{12}$ is not a higher order polynomial in momenta, as typically found using the Lax pair technique in similar integrable models (such as the Toda chain [52]). We expect that also at $N>3$ the ansatz (20) leads to $N-2$ additional independent charges in involution.

\section{FUTURE DIRECTIONS AND RELATION TO $T \bar{T}$}

The presented results open numerous avenues for future research. In particular, what about the quantum integrability of (18)? The following argument indicates that it should hold. The classical two-particle phase shift following from (18) is $\delta=s$, which coincides with the exact phase shift of a known quantum integrable model-the $T \bar{T}$ deformation of a free massless fermion [19,53-56] (see, e.g., [57,58] for explicit derivations of the corresponding classical action). This indicates that there exists a quantization of (18) resulting in the same phase shift, and that (18) describes the $N$-particle subsector of the $T \bar{T}$ deformed fermion, similarly to how the Ruijsenaars-Schneider model describes the $N$-particle subsector of the sine-Gordon theory [48]. The appearance of the shadow Poincaré subalgebra in (22) mirrors the presence of dynamical and world sheet "clocks and rods" in the gravitational formulation of the $T \bar{T}$ deformation [59-62]. This relation also suggests that the infinite quon Hilbert space $\mathcal{H}_{w}$ may provide a natural arena for defining off-shell observables in $T \bar{T}$ deformed theories. It will be interesting to connect this to the recent construction of [63]. Finally, this relation indicates that an integrable relativistic $N$-body system described here is actually a model of dynamical geometry (gravity), see also [64].

As far as the $D=2$ QCD physics goes, it was noticed in numerical studies of the spectrum that meson mass eigenstates have definite parton numbers with a very high accuracy even for small quark masses [34,35]. It was also observed that the equation for the spectrum becomes exactly solvable in the high energy limit [36]. Both these observations should be related to the integrability found here and it will be useful to make the connection precise.

In addition, it will be very interesting to see which of the presented results can be generalized to $D=3,4$ and to connect this approach to other signs of approximate integrability in QCD, such as $[65,66]$. It is encouraging to see that an integrable $T \bar{T}$-deformed model emerged at high energies in the $D=2$ case, given that according to the ASA the integrable models appearing at $D=3,4$ are also $T \bar{T}$ deformations.

\section{ACKNOWLEDGMENTS}

We thank Misha Feigin, Vitya Gorbenko, Sam Grushevsky, Misha Ivanov, Igor Klebanov, Zohar Komargodski, Grisha Korchemsky, Conghuan Luo and Riccardo Rattazzi for useful discussions. This work is supported in part by the NSF CAREER Grant No. PHY-1352119. 
[1] G. 't Hooft, A planar diagram theory for strong interactions, Nucl. Phys. B72, 461 (1974).

[2] J. M. Maldacena, The large N limit of superconformal field theories and supergravity, Adv. Theor. Math. Phys. 2, 231 (1998).

[3] N. Beisert et al., Review of AdS/CFT integrability: An overview, Lett. Math. Phys. 99, 3 (2012).

[4] S. Dubovsky and V. Gorbenko, Towards a theory of the QCD string, J. High Energy Phys. 02 (2016) 022.

[5] S. Dubovsky, R. Flauger, and V. Gorbenko, Effective string theory revisited, J. High Energy Phys. 09 (2012) 044.

[6] P. Cooper, S. Dubovsky, V. Gorbenko, A. Mohsen, and S. Storace, Looking for integrability on the world sheet of confining strings, J. High Energy Phys. 04 (2015) 127.

[7] J. Polchinski and A. Strominger, Effective String Theory, Phys. Rev. Lett. 67, 1681 (1991).

[8] A. Athenodorou, B. Bringoltz, and M. Teper, Closed flux tubes and their string description in $D=3+1 \mathrm{SU}(\mathrm{N})$ gauge theories, J. High Energy Phys. 02 (2011) 030.

[9] A. Athenodorou, B. Bringoltz, and M. Teper, Closed flux tubes and their string description in $D=2+1 \mathrm{SU}(\mathrm{N})$ gauge theories, J. High Energy Phys. 05 (2011) 042.

[10] A. Athenodorou and M. Teper, Closed flux tubes in higher representations and their string description in $D=2+1 \mathrm{SU}(\mathrm{N})$ gauge theories, J. High Energy Phys. 06 (2013) 053.

[11] A. Athenodorou and M. Teper, Closed flux tubes in $D=2+1 \mathrm{SU}(\mathrm{N})$ gauge theories: Dynamics and effective string description, J. High Energy Phys. 10 (2016) 093.

[12] A. Athenodorou and M. Teper, SU(N) gauge theories in $2+1$ dimensions: Glueball spectra and $k$-string tensions, J. High Energy Phys. 02 (2017) 015.

[13] A. Athenodorou and M. Teper, On the mass of the worldsheet "axion" in $S U(N)$ gauge theories in $3+1$ dimensions, Phys. Lett. B 771, 408 (2017).

[14] M. Teper, Large $N$ and confining flux tubes as stringsA view from the lattice, Acta Phys. Pol. B 40, 3249 (2009).

[15] B. Lucini and M. Panero, SU(N) gauge theories at large N, Phys. Rep. 526, 93 (2013).

[16] S. Dubovsky, R. Flauger, and V. Gorbenko, Flux tube spectra from approximate integrability at low energies, J. Exp. Theor. Phys. 120, 399 (2015).

[17] S. Dubovsky and G. Hernández-Chifflet, Yang-Mills glueballs as closed bosonic strings, J. High Energy Phys. 02 (2017) 022.

[18] C. Chen, P. Conkey, S. Dubovsky, and G. HernándezChifflet, Undressing confining flux tubes with $T \bar{T}$, Phys. Rev. D 98, 114024 (2018).

[19] S. Dubovsky, R. Flauger, and V. Gorbenko, Solving the simplest theory of quantum gravity, J. High Energy Phys. 09 (2012) 133.

[20] S. Dubovsky, A simple world sheet black hole, J. High Energy Phys. 07 (2018) 011.

[21] M. F. Paulos, J. Penedones, J. Toledo, B. C. van Rees, and P. Vieira, The $S$-matrix bootstrap. Part I: QFT in AdS, J. High Energy Phys. 11 (2017) 133.

[22] M. F. Paulos, J. Penedones, J. Toledo, B. C. van Rees, and P. Vieira, The $S$-matrix bootstrap II: Two dimensional amplitudes, J. High Energy Phys. 11 (2017) 143.
[23] D. Poland, S. Rychkov, and A. Vichi, The conformal bootstrap: Theory, numerical techniques, and applications, Rev. Mod. Phys. 91, 015002 (2019).

[24] J. E. Miro, A. L. Guerrieri, A. Hebbar, J. Penedones, and P. Vieira, Flux Tube $S$-Matrix Bootstrap, Phys. Rev. Lett. 123, 221602 (2019).

[25] S. Dubovsky, R. Flauger, and V. Gorbenko, Evidence for a New Particle on the World Sheet of the QCD Flux Tube, Phys. Rev. Lett. 111, 062006 (2013).

[26] J. C. Donahue, S. Dubovsky, G. Hernández-Chifflet, and S. Monin, From QCD strings to WZW, J. High Energy Phys. 03 (2019) 120.

[27] S. Dubovsky, The QCD $\beta$-function on the string world sheet, Phys. Rev. D 98, 114025 (2018).

[28] M. Luscher, Symmetry breaking aspects of the roughening transition in gauge theories, Nucl. Phys. B180, 317 (1981).

[29] M. Luscher and P. Weisz, String excitation energies in SU(N) gauge theories beyond the free-string approximation, J. High Energy Phys. 07 (2004) 014.

[30] O. Aharony and N. Klinghoffer, Corrections to NambuGoto energy levels from the effective string action, J. High Energy Phys. 12 (2010) 058.

[31] O. Aharony and M. Field, On the effective theory of long open strings, J. High Energy Phys. 01 (2011) 065.

[32] O. Aharony and Z. Komargodski, The effective theory of long strings, J. High Energy Phys. 05 (2013) 118.

[33] A. Armoni, Y. Frishman, and J. Sonnenschein, The string tension in two-dimensional gauge theories, Int. J. Mod. Phys. A 14, 2475 (1999).

[34] S. Dalley and I. R. Klebanov, String spectrum of $(1+1)-$ dimensional large $N$ QCD with adjoint matter, Phys. Rev. D 47, 2517 (1993).

[35] G. Bhanot, K. Demeterfi, and I. R. Klebanov, 1+1)dimensional large $N$ QCD coupled to adjoint fermions, Phys. Rev. D 48, 4980 (1993).

[36] D. Kutasov, Two-dimensional QCD coupled to adjoint matter and string theory, Nucl. Phys. B414, 33 (1994).

[37] E. Katz, G. M. Tavares, and Y. Xu, Solving 2D QCD with an adjoint fermion analytically, J. High Energy Phys. 05 (2014) 143.

[38] S. R. Coleman, More about the massive Schwinger model, Ann. Phys. (N.Y.) 101, 239 (1976).

[39] E. Witten, $\theta$ vacua in two-dimensional quantum chromodynamics, Nuovo Cimento A 51, 325 (1979).

[40] A. M. Polyakov, String theory and quark confinement, Nucl. Phys. B, Proc. Suppl. 68, 1 (1998).

[41] O. W. Greenberg, Example of Infinite Statistics, Phys. Rev. Lett. 64, 705 (1990).

[42] S. Weinberg, The Quantum Theory of Fields. Vol. 1: Foundations (Cambridge University Press, Cambridge, England, 2005).

[43] J. C. Donahue and S. Dubovsky (to be published).

[44] E. Karat, An example of Poincare symmetry with a central charge, Phys. Lett. B 445, 337 (1999).

[45] D. G. Currie, T. F. Jordan, and E. C. G. Sudarshan, Relativistic invariance and Hamiltonian theories of interacting particles, Rev. Mod. Phys. 35, 350 (1963).

[46] D. Currie, Interaction contra classical relativistic Hamiltonian particle mechanics, J. Math. Phys. (N.Y.) 4, 1470 (1963). 
[47] H. Leutwyler, A no-interaction theorem in classical relativistic Hamiltonian particle mechanics, Il Nuovo Cimento (1955-1965) 37, 556 (1965).

[48] S. N. M. Ruijsenaars and H. Schneider, A new class of integrable systems and its relation to solitons, Ann. Phys. (N.Y.) 170, 370 (1986).

[49] S. N. M. Ruijsenaars, Relativistic Toda systems, Commun. Math. Phys. 133, 217 (1990).

[50] S. Lenz and B. Schreiber, Example of a Poincare anomaly in relativistic quantum mechanics, Phys. Rev. D 53, 960 (1996).

[51] Yu. S. Kalashnikova and A. V. Nefediev, 1+1) string with quarks at the ends revisited, Phys. Lett. B 399, 274 (1997).

[52] V. Bazhanov, P. Dorey, K. Kajiwara, and K. Takasaki, Call for papers: Special issue on fifty years of the Toda lattice, J. Phys. A 50, 310201 (2017).

[53] A. B. Zamolodchikov, Expectation value of composite field $\mathrm{T}$ anti-T in two-dimensional quantum field theory, arXiv: hep-th/0401146.

[54] S. Dubovsky, V. Gorbenko, and M. Mirbabayi, Natural tuning: Towards a proof of concept, J. High Energy Phys. 09 (2013) 045.

[55] F. A. Smirnov and A. B. Zamolodchikov, On space of integrable quantum field theories, Nucl. Phys. B915, 363 (2017).

[56] A. Cavaglià, S. Negro, I. M. Szécsényi, and R. Tateo, $T \bar{T}$ deformed 2D quantum field theories, J. High Energy Phys. 10 (2016) 112.
[57] G. Bonelli, N. Doroud, and M. Zhu, $T \bar{T}$-deformations in closed form, J. High Energy Phys. 06 (2018) 149.

[58] E. A. Coleman, J. Aguilera-Damia, D. Z. Freedman, and R. M. Soni, $T \bar{T}$-deformed actions and $(1,1)$ supersymmetry, J. High Energy Phys. 10 (2019) 080.

[59] S. Dubovsky, V. Gorbenko, and M. Mirbabayi, Asymptotic fragility, near $\mathrm{AdS}_{2}$ holography and $T \bar{T}$, J. High Energy Phys. 09 (2017) 136.

[60] J. Cardy, The $T \bar{T}$ deformation of quantum field theory as random geometry, J. High Energy Phys. 10 (2018) 186.

[61] S. Dubovsky, V. Gorbenko, and G. Hernández-Chifflet, $T \bar{T}$ partition function from topological gravity, J. High Energy Phys. 09 (2018) 158.

[62] R. Conti, S. Negro, and R. Tateo, The TT̄ perturbation and its geometric interpretation, J. High Energy Phys. 02 (2019) 085.

[63] J. Cardy, $T \bar{T}$ deformation of correlation functions, J. High Energy Phys. 12 (2019) 160.

[64] S. Dubovsky, Geometry from confinement, in Proceedings of the KITP Conference: Geometry from the Quantum, 2020, http://online.kitp.ucsb.edu/online/qgravity_ c20/dubovsky/.

[65] G. Ferretti, R. Heise, and K. Zarembo, New integrable structures in large-N QCD, Phys. Rev. D 70, 074024 (2004).

[66] N. Beisert, G. Ferretti, R. Heise, and K. Zarembo, One-loop QCD spin chain and its spectrum, Nucl. Phys. B717, 137 (2005). 